\title{
Synthesis of Novel Pyrimido Thiazine and Their Derivatives
}

\section{Sirsat Shivraj Balajirao*, Jadhav Anilkumar Govindrao, Kale Prashant Sambharao, Jadhav Madhav Sopanrao}

Post Graduate Research Centre, Department of Chemistry, Yeshwant Mahavidyalaya, Nanded, India

Email address:

sbs.igm@gmail.com (S. S. Balajirao)

${ }^{*}$ Corresponding author

\section{To cite this article:}

Sirsat Shivraj Balajirao, Jadhav Anilkumar Govindrao, Kale Prashant Sambharao, Jadhav Madhav Sopanrao. Synthesis of Novel Pyrimido Thiazine and Their Derivatives. American Journal of Heterocyclic Chemistry. Vol. 5, No. 2, 2019, pp. 23-26.

doi: 10.11648/j.ajhc.20190502.11

Received: May 18, 2019; Accepted: June 19, 2019; Published: June 29, 2019

\begin{abstract}
In present report novel thiazines were prepared from starting materials chalcone and thiourea. The resulting compound 6-(4-methoxyphenyl) 4-phenyl-6H-1, 3-thiazin-2-amine (2) was further reacted with ethyl 2-cyano-3, 3-bis (methylthio) acrylate in the presence of catalytic amount of potassium carbonate in DMF under reflux condition that offered novel 2-(4-methoxyphenyl) 8-(methylthio)-6-0xo-4-phenyl-4, 6, 9, 9a-tetrahydropyrimido [2, 1-b] [1, 3]-thiazine-7-carbonitrile (3). The synthesized compounds were characterized by spectral methods. The compound (3) possesses replaceable methylthio $\left(-\mathrm{SCH}_{3}\right)$ group at 8 position. The compound (3) react with various nucleophiles like substituted aromatic amines, aromatic phenols, hetryl amines and active methylene compounds to give 2-(4-methoxyphenyl)-8-substituted-6-oxo-4-phenyl-4, 6, 9, 9a-tetrahydropyrimido $[2,1-\mathrm{b}][1,3]$ thiazine-7-carbonitrile in good yields.
\end{abstract}

Keywords: Claisen-Schmidt Condensation, 2-Cyano-3, 3-Bis (Methylthio) Acrylate,Michael Addition Reaction, Thiazines

\section{Introduction}

Thiazines are group of heterocyclic organic compounds that are still largely unexplored for their pharmacological activities. It is commonly reported that heterocyclic thiazine derivatives with nitrogen and sulphur are important because they are biological constituent of many biomolecules and drugs [1]. Thiazines and it's derivatives have been reported to exhibit a variety of biological activities like Antibacterial [2], Antifungal [3], Antitubercular [4], Anti-inflammatory [5], Analgesic- [6], Sedative-hypnotic [7], Immunosuppressive agents [8], Herbicidal [9], Antihistamin- ic [10], Anti-HIV activities [11], Antiproliferative [12]. These are also known as anti-radiation agents and used as radiation-sickness drugs [13]. In the view of this observation and extension of earlier work, we have synthesized novel 2-(4-methoxyphenyl)8(methylthio)-6-oxo-4-phenyl-4, 6, 9, 9a-tetrahydropyrimido $[2,1-b][1,3]$-thiazine-7-carbonitrile by using 3-(4methoxyphenyl)-1-phenyl prop-2-en-1-one [14-15] and 6-(4methoxyphenyl) 4-phenyl-6H-1, 3-thiazin-2-amine [16-17].

\section{Methods}

Melting points were determined in open capillary tubes and are uncorrected. The silica gel $\mathrm{F}_{254}$ plates were used for thin layer chromatography (TLC); the spots were examined under UV light and then developed in an iodine vapor. Column chromatography was performed with silica gel (BDH 100-200 mesh). Solvents were purified according to standard procedures. The spectra were recorded as follows: IR, $\mathrm{KBr}$ pellets, a Perkin-Elmer RX1 FT-IR spectrophotometer; ${ }^{1} \mathrm{H} \mathrm{NMR}, \mathrm{CDCl}_{3}, 200 \mathrm{MHz}$, a Varian Gemini 200 instrument. Elemental analysis was performed on a Heraeus CHN-O rapid analyzer.

\subsection{2-(4-methoxyphenyl)-8 (methylthio)-6-0xo-4-phenyl-4, 6, 9, 9a-tetrahydro pyrimido- $[2,1-b][1,3]$ thiazine-7- carbonitrile}

\section{Step $-I$}

A solution of $\mathrm{KOH} 50 \%$ is added to an equimolar solution of acetophenone (0.01 mole) and 4-methoxybenzaldehyde ( 0.01 mole) in ethanol $95 \%$; the addition is performed under energetic stirring at room temperature. The reaction is left 
under stirring for one night and then diluted with water and acidified; the precipitate is separated by filtration and dried under vacuum. They are crystallized by ethanol compound.

Step - II

A mixture of chalcone i.e. 3-(4-methoxyphenyl)-1phenylprop-2-en-1-one (2.38 gm, 0.01 mole ), and thiourea (0.76 gm., 0.01 mole ) were dissolved in ethanoic potassium hydroxide solution $(10 \mathrm{ml})$ was heated for $4 \mathrm{hrs}$., then it was poured into cold ice obtained 6-(4-methoxyphenyl)-4-phenyl$6 \mathrm{H}-1,3$-thiazin-2-amine (2).

Step - III

A mixture of 6-(4-methoxyphenyl)-4-phenyl-6H-1, 3thiazin-2-amine (2) and ethyl 2-Cyano-3, 3-bis (methylthio) acrylate in the presence of catalytic amount of potassium carbonate $(10 \mathrm{mg})$ in DMF was refluxed for 4 hours, the reaction was monitored by TLC. After completion, the reaction mixture was cooled at room temperature then wash with water the extracted with ethyl acetate. The extract was concentrated and the residue was subjected to column chromatography (silica gel, n-hexane-ethyl acetate $8: 2$ ) to obtain pure solid compound 2-(4-methoxyphenyl)-8(methylthio)-6-oxo-4-phenyl-4, 6, 9, 9a-tetrahydropyrimido $[2,1-b][1,3]$ thiazine-7-carbonitrile (3). The compound (3) confirmed by IR, ${ }^{1} \mathrm{H}$ and $\mathrm{C}^{13} \mathrm{NMR}$ and MS analytical data.

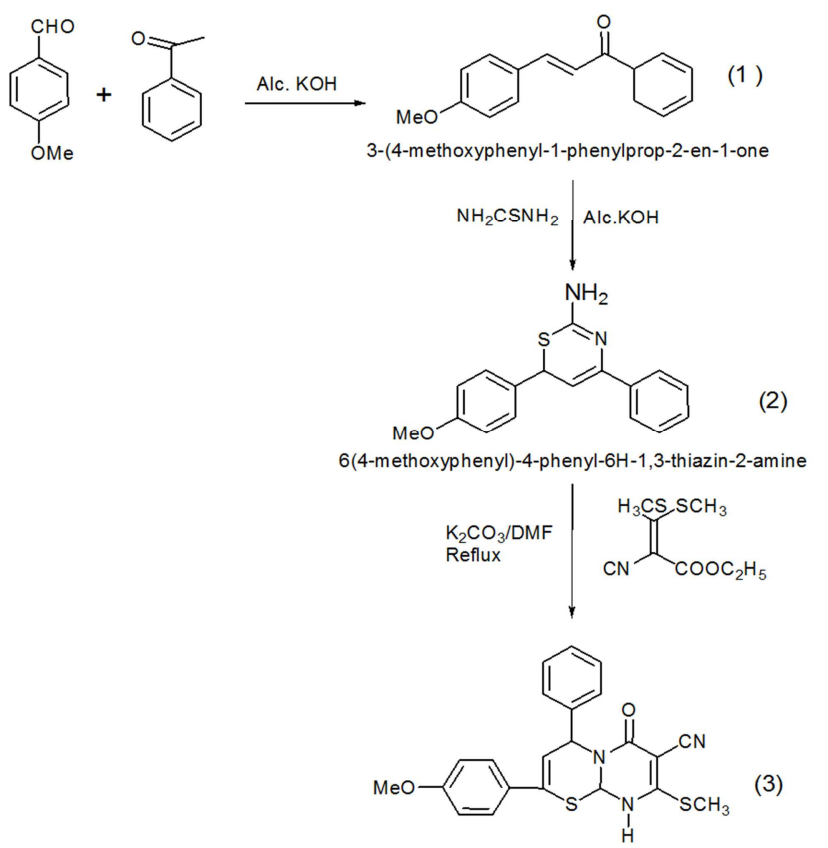

Figure 1. 2-(4-methoxyphenyl)-8-(methylthio)-6-oxo-4-phenyl-4, 6, 9, 9atetrahydropyrimido $[2,1-b][1,3]$ thiazine-7-carbonitrile.

\subsection{Synthesis of Derivatives}

A mixture of (3) (1mmol) and independently, various substituted aromatic amines, aromatic phenols, heteryl amines and active methylene compounds $(1 \mathrm{mmol})$ in DMF $(10 \mathrm{ml})$ and anhydrous potassium carbonate $(10 \mathrm{mg})$ was reflux for 4 to $6 \mathrm{hrs}$. The reaction mixture cooled to room temperature and poured into ice cold water. The separated solid product was filtered, washed with water and recrystallised using ethyl alcohol.
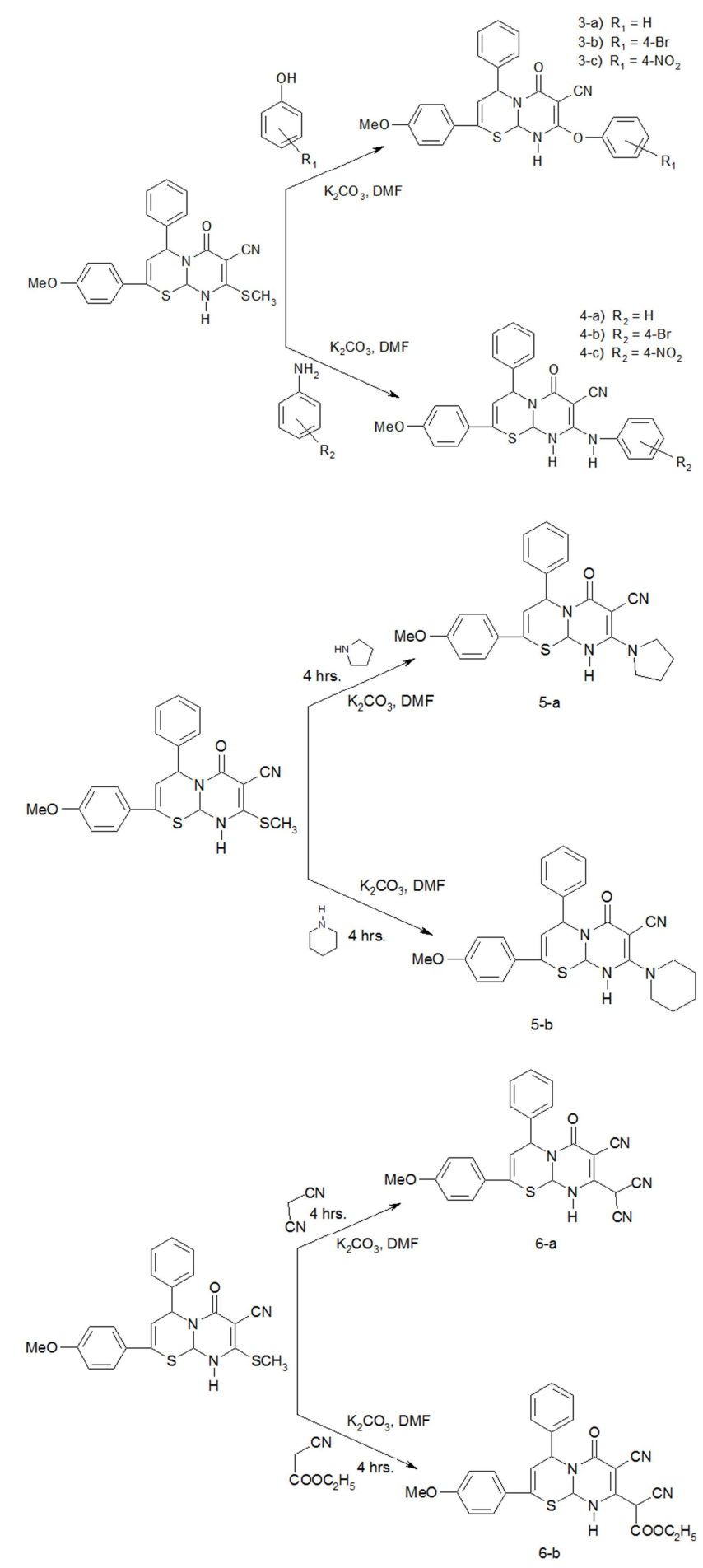

Figure 2. 2-(4-methoxyphenyl)-8-(substituted)-6-oxo-4-phenyl-4,6,9,9atetrahydropyrimido[2,1-b][1,3]thiazine-7-carbonitrile.

\section{Result and Discussion}

The compound 2-(4-methoxyphenyl)-8-(methylthio)-60xo-4-phenyl-4, 6, 9, 9a-tetrahydropyrimido [2, 1-b] [1, 3] thiazine-7-carbonitrile are synthesized by dissolving 6-(4methoxyphenyl)4-phenyl-6H-1, 3-thiazine-2-amine (2) and ethyl 2-cyano-3-3-bis (methylthio) acrylate in the presence of 
$\mathrm{K}_{2} \mathrm{CO}_{3}$ in DMF under reflux condition. The synthesized compound acts as electrophilic species reacting with various substituted aromatic amines, aromatic phenols, hetryl amines and active methylene compound gives 2-(4-methoxyphenyl)8-substituted-6-oxo-4-phenyl-4, 6, 9, 9a-tetrahydropyrimido $[2,1, \mathrm{~b}][1,3]$ thiazine-7-carbonitrile in good yields.

\section{Conclusion}

A new different 2-(4-methoxyphenyl)-8-(methylthio)-6oxo-4-phenyl-4, 6, 9, 9 a-tetrahydropyrimido [2, 1-b] [1, 3] thiazine-7-carbonitrile are synthesized by using simple and efficient chemistry and this synthesized compounds possesses methylthio group at 8-position which is best leaving group therefore synthesized compound act as an electrophilic species and reacting with various nucleophiles. In compound (3) cyano and thiomethyl groups are at adjacent position it also undergo cyclization to give polycyclic heterocyclic compound.

3) 2-(4-methoxyphenyl)-8(methylthio)-6oxo-4-phenyl-4, 6, 9, 9atetrahydro-pyrimido $[2,1-\mathrm{b}][1,3]$ thiazine-7-carbonitrile.

IR: 3330, 2210, 1650, $1050 \mathrm{~cm}^{-1}$;

${ }^{1} \mathrm{H}$ NMR: $\delta 2.40\left(\mathrm{~s}, 3 \mathrm{H}, \mathrm{SCH}_{3}\right), 5.10(\mathrm{~s}, 1 \mathrm{H} \mathrm{NH}), 6.48(\mathrm{~s}, 1 \mathrm{H}$ $=\mathrm{CH}) 5.74(\mathrm{~s} 1 \mathrm{H} \mathrm{CH}), 5.50(\mathrm{~s}, 1 \mathrm{H} \mathrm{CH}), 7.18(\mathrm{~s}, 5 \mathrm{H} \mathrm{Ar}-\mathrm{H}), 7.20(\mathrm{dd}$, 2H Ar-H), 6.95 (dd, 2H Ar-H), 3.76 (s, 3H).

ESI-MS: 421.

Anal. Calcd for $\mathrm{C}_{22} \mathrm{H}_{19} \mathrm{~N}_{3} \mathrm{O}_{2} \mathrm{~S}_{2}:$ C, 62.68; H, 4.54; N, 9.97; O, 7.59; S, 15.21

Found: C, 62.63; H, 4.52; N, 9.96; O, 7.63; S, 15.26.

Mol. Formula: $\mathrm{C}_{22} \mathrm{H}_{19} \mathrm{~N}_{3} \mathrm{O}_{2} \mathrm{~S}_{2}$

Mol. Wt.: 421.

3-a) 2-(4-methoxyphenyl)-6-0xo-8-phenoxy-4-phenyl-4, 6, 9, 9atetrahydropyri- mido-[2, 1-b] [1,3]thiazine-7-carbonitrile.

IR: 3330, 2210, 1650, 1050, $\mathrm{cm}^{-1}$;

${ }^{1} \mathrm{H}$ NMR: $5.10(\mathrm{~s}, 1 \mathrm{H} \mathrm{NH}), 6.47(\mathrm{~s}, 1 \mathrm{H}=\mathrm{CH}) 5.75(\mathrm{~s}, 1 \mathrm{H} \mathrm{CH})$, 5.52 (s, 1H CH), 7.24 (s, 5H Ar-H), 6.90 (dd, 2H Ar-H), 7.21 (dd, 2H Ar-H), 7.05 (s 5H Ar-H), 3.80 (s, 3H)

ESI-MS: 454.

Anal. Calcd: $\mathrm{C}_{27} \mathrm{H}_{21} \mathrm{~N}_{3} \mathrm{O}_{3} \mathrm{~S}$ C, 69.36; H, 4.53; N, 8.99; O, 10.27; S, 6.86

Found: C, 69.34; H, 4.52; N, 8.98; O, 10.28; S, 6.88.

Mol. Formula: $\mathrm{C}_{27} \mathrm{H}_{21} \mathrm{~N}_{3} \mathrm{O}_{3} \mathrm{~S}$

Mol. Wt.:454.

3-b) 8-(4-bromophenoxy)-2-(4-methoxyphenyl)-6-oxo-4-phenyl4, 6, 9, 9a-tetra-hydropyrimido [2, 1-b] [1, 3] thiazine-7carbonitrile.

IR: 3330, 2210, 1650, 1050, $660 \mathrm{~cm}^{-1}$;

${ }^{1} \mathrm{H}$ NMR: 5.11(s, $\left.1 \mathrm{H} \mathrm{NH}\right), 6.46(\mathrm{~s}, 1 \mathrm{H}=\mathrm{CH}) 5.73(\mathrm{~s}, 1 \mathrm{H} \mathrm{CH})$, 5.54 (s, 1H CH), 7.19 (s, 5H Ar-H), 6.94 (dd, 2H Ar-H), 7.23 (dd, 2H Ar-H), 6.88 (dd 2H Ar-H), 7.24 (dd 2 H Ar-H ), 3.78 (s, 3H)

ESI-MS: 545.

Anal. Calcd: $\mathrm{C}_{27} \mathrm{H}_{20} \mathrm{BrN}_{3} \mathrm{O}_{3} \mathrm{~S}: \mathrm{C}, 59.35 ; \mathrm{H}, 3.69 ; \mathrm{Br}, 14.62 ; \mathrm{N}$, 7.69; O, 8.78; S, 5.87.

Found: C, 59.31; H, 3.62; Br, 14.60; N, 7.71; O, 8.83; S, 5.93.

Mol. Formula: $\mathrm{C}_{27} \mathrm{H}_{20} \mathrm{BrN}_{3} \mathrm{O}_{3} \mathrm{~S}$

Mol. Wt: 545.

3-c) 2-(4-methoxyphenyl)-8-(4-nitrophenoxy)-6-oxo-4-phenyl-4,
6, 9, 9a-tetrahy-dropyrimido $[2,1-b][1,3]$ thiazine-7-carbonitrile. IR: 3330, 2210, 1650, 1050, $1510 \mathrm{~cm}^{-1}$;

${ }^{1} \mathrm{H}$ NMR: $5.09(\mathrm{~s}, 1 \mathrm{H} \mathrm{NH}), 6.49(\mathrm{~s}, 1 \mathrm{H}=\mathrm{CH}) 5.76(\mathrm{~s}, 1 \mathrm{H} \mathrm{CH})$, 5.53 (s, 1H CH), 7.22 (s, 5H Ar-H), 6.92 (dd, 2H Ar-H), 7.23 (dd, 2H Ar-H), 7.18 (dd 2 H Ar-H), 7.96 (dd 2 H Ar-H ), 3.76 (s, 3H) ESI-MS: 512.

Anal. Calcd: $\mathrm{C}_{27} \mathrm{H}_{20} \mathrm{~N}_{4} \mathrm{O}_{5} \mathrm{~S}$ : C, 63.27; H, 3.93; N, 10.93; O, 15.61; S, 6.26. Found: C, 63.29; H, 3.91; N, 10.91; O, 15.62, S, 6.27.

Mol. Formula: $\mathrm{C}_{27} \mathrm{H}_{20} \mathrm{~N}_{4} \mathrm{O}_{5} \mathrm{~S}$

Mol. Wt.: 512.

4-a) 2-(4-Methoxyphenyl)-6-oxo-4-phenyl-8-(phenylamino)-4, 6, 9, 9a-tetrahy-dropyrimido [2, 1-b] [1,3] thiazine-7-carbonitrile.

IR: $3330,2210,1650,1050,1590 \mathrm{~cm}^{-1}$;

${ }^{1} \mathrm{H}$ NMR: $5.10(\mathrm{~s}, 1 \mathrm{H} \mathrm{NH}), 4.10(\mathrm{~s}, 1 \mathrm{NH}), 6.43(\mathrm{~s}, 1 \mathrm{H}=\mathrm{CH}) 5.75$ (s, 1H CH), 5.54 (s, 1H CH), 7.20 (s, 5H Ar-H), 6.89 (dd, 2H Ar-H), 7.25 (dd, 2H Ar-H), 7.01 (s 5H Ar-H), 3.80 (s, 3H).

ESI-MS: 466.

Anal. Calcd: $\mathrm{C}_{27} \mathrm{H}_{22} \mathrm{~N}_{4} \mathrm{O}_{2} \mathrm{~S}: \mathrm{C}, 69.51 ; \mathrm{H}, 4.75 ; \mathrm{N}, 12.01 ; \mathrm{O}, 6.86$;

S, 6.87 Found: C, 69.48; H, 4.81; N, 12.03; O, 6.83; S, 6.85

Mol. Formula: $\mathrm{C}_{27} \mathrm{H}_{22} \mathrm{~N}_{4} \mathrm{O}_{2} \mathrm{~S}$

Mol. Wt.: 466.

4-b) 8-((4-bromophenyl)amino)-2-(4-methoxyphenyl)-6-oxo-4phenyl-4, 6, 9, 9a-tetrahydropyrimido $[2,1-\mathrm{b}][1,3]$ thiazine-7carbonitrile.

IR: 3330, 2210, 1650, 1050, $660 \mathrm{~cm}^{-1}$;

${ }^{1} \mathrm{H}$ NMR: $5.08(\mathrm{~s}, 1 \mathrm{H} \mathrm{NH}), 4.13(\mathrm{~s}, 1 \mathrm{NH}), 6.46(\mathrm{~s}, 1 \mathrm{H}=\mathrm{CH}) 5.76$ (s, 1H CH), $5.50(\mathrm{~s}, 1 \mathrm{H} \mathrm{CH}), 7.22$ (s, 5H Ar-H), 6.92 (dd, 2H Ar-H), 7.23 (dd, 2H Ar-H), 6.49 (dd 2H Ar-H), 7.32 (dd 2 H Ar-H), 3.78 (s, $3 \mathrm{H})$

ESI-MS: 545

Anal. Calcd for $\mathrm{C}_{27} \mathrm{H}_{21} \mathrm{BrN}_{4} \mathrm{O}_{2} \mathrm{~S}$ : C, 59.45; H, 3.88; $\mathrm{Br}, 14.65 ; \mathrm{N}$, 10.27; O, 5.87; S, 5.88

Found: C, 59.42; H, 3.91; Br, 14.58; N, 10.32; O, 5.89; S, 5.88

Mol. Formula: $\mathrm{C}_{27} \mathrm{H}_{21} \mathrm{BrN}_{4} \mathrm{O}_{2} \mathrm{~S}$,

Mol. Wt.: 545.

4-c) 2-(4-methoxyphenyl)-8-((4-nitrophenyl)amino)-6-oxo-4phenyl-4, 6, 9, 9a-tetrahydropyrimido $[2,1-b][1,3]$ thiazine-7carbonitrile.

IR: 3330, 2210, 1650, 1050, $1580 \mathrm{~cm}^{-1}$;

${ }^{1} \mathrm{H}$ NMR: 5.11 (s, 1H NH), $4.09(\mathrm{~s}, 1 \mathrm{NH}), 6.44(\mathrm{~s}, 1 \mathrm{H}=\mathrm{CH}) 5.72$ (s, 1H CH), 5.56 (s, 1H CH), 7.21 (s, 5H Ar-H), 6.88 (dd, 2H Ar-H), 7.25 (dd, 2H Ar-H), 6.63 (dd 2H Ar-H), 7.98 (dd 2H Ar-H), 3.80 (s, $3 \mathrm{H})$

ESI-MS: 511.

Anal. Calcd: $\mathrm{C}_{27} \mathrm{H}_{21} \mathrm{~N}_{5} \mathrm{O}_{4} \mathrm{~S}: \mathrm{C}, 63.39 ; \mathrm{H}, 4.14 ; \mathrm{N}, 13.69 ; \mathrm{O}, 12.51$; S, 6.27 Found: C, 63.45; H, 4.13; N, 13.67; O, 12.48; S, 6.27.

Mol. Formula: $\mathrm{C}_{27} \mathrm{H}_{21} \mathrm{~N}_{5} \mathrm{O}_{4} \mathrm{~S}$.

Mol. Wt.: 511.

5-a) 2-(4-methoxyphenyl)-6-oxo-4-phenyl-8-(pyrrolidin-1-yl) 4, 6, 9, 9a-tetrahy-dropyrimido [2, 1-b] [1,3] thiazine-7-carbonitrile: IR: $3330,2210,1650,1050,1580 \mathrm{~cm}^{-1}$;

${ }^{1} \mathrm{H}$ NMR: $5.10(\mathrm{~s}, 1 \mathrm{H} \mathrm{NH}), 6.45(\mathrm{~s}, 1 \mathrm{H}=\mathrm{CH}) 5.76(\mathrm{~s}, 1 \mathrm{H} \mathrm{CH})$, 5.52 (s, 1H CH), 7.22 (s, 5H Ar-H), 6.90 (dd, 2H Ar-H), 7.23 (dd, $2 \mathrm{H} \mathrm{Ar}-\mathrm{H}), 3.81(\mathrm{~s}, 3 \mathrm{H}), 2.60(\mathrm{t}, 4 \mathrm{H}), 1.65(\mathrm{~m}, 4 \mathrm{H})$

ESI-MS: 444.

Anal. Calcd: $\mathrm{C}_{25} \mathrm{H}_{24} \mathrm{~N}_{4} \mathrm{O}_{2} \mathrm{~S}, \mathrm{C}, 67.54 ; \mathrm{H}, 5.44 ; \mathrm{N}, 12.60 ; \mathrm{O}, 7.20$; S, 7.21. Found: C, 67.57; H, 5.41; N, 12.56; O, 7.23; S, 7.23 
Mol. Formula: $\mathrm{C}_{25} \mathrm{H}_{24} \mathrm{~N}_{4} \mathrm{O}_{2} \mathrm{~S}$

Mol. Wt.: 444

5-b) 2-(4-methoxyphenyl)-6-oxo-4-phenyl-8-(piperidin-1-yl)-4, 6, 9, 9a-tetrahy-dropyrimido [2, 1-b] [1,3] thiazine-7-carbonitrile: IR: 3330, 2210, 1650, 1050, $1580 \mathrm{~cm}^{-1}$;

${ }^{1} \mathrm{H}$ NMR: $5.13(\mathrm{~s}, 1 \mathrm{H} \mathrm{NH}), 6.48(\mathrm{~s}, 1 \mathrm{H}=\mathrm{CH}) 5.72(\mathrm{~s}, 1 \mathrm{H} \mathrm{CH})$, 5.54 (s, 1H CH), 7.20 (s, 5H Ar-H), $6.86(\mathrm{dd}, 2 \mathrm{H} \mathrm{Ar}-\mathrm{H}), 7.23$ (dd, $2 \mathrm{H} \mathrm{Ar}-\mathrm{H}), 3.77$ (s, 3H), $3.13(\mathrm{t}, 4 \mathrm{H}), 1.53(\mathrm{~m}, 6 \mathrm{H})$

ESI-MS: 458.

Anal. Calcd: $\mathrm{C}_{26} \mathrm{H}_{26} \mathrm{~N}_{4} \mathrm{O}_{2} \mathrm{~S}, \mathrm{C}, 68.10 ; \mathrm{H}, 5.71 ; \mathrm{N}, 12.22 ; \mathrm{O}, 6.98$; S, 6.99 .

Found: C, 68.18; H, 5.69; N, 12.22; O, 6.94; S, 6.97.

Mol. Formula: $\mathrm{C}_{26} \mathrm{H}_{26} \mathrm{~N}_{4} \mathrm{O}_{2} \mathrm{~S}$.

Mol. Wt.: 458.

6-a) 2-[7-Cyano-2-(4-methoxyphenyl)-6-oxo-4-phenyl-4, 6, 9, 9a-tetrahydropyri-mido [2, 1-b] [1,3] thiazin-8-yl]-malononitrile:

IR: 3330, 2210, 1650, $1050 \mathrm{~cm}^{-1}$;

${ }^{1} \mathrm{H}$ NMR: $5.10(\mathrm{~s}, 1 \mathrm{H} \mathrm{NH}), 6.49(\mathrm{~s}, 1 \mathrm{H}=\mathrm{CH}) 5.73(\mathrm{~s}, 1 \mathrm{H} \mathrm{CH})$, 5.51 (s, 1H CH), 7.23 (s, 5H Ar-H), 6.91 (dd, 2H Ar-H), 7.22 (dd,

$2 \mathrm{H} \mathrm{Ar}-\mathrm{H}), 4.16$ (s $1 \mathrm{H}$ act.-CH), 3.81 (s, 3H)

ESI-MS: 439.

Anal. Calcd: $\mathrm{C}_{24} \mathrm{H}_{17} \mathrm{~N}_{5} \mathrm{O}_{2} \mathrm{~S}, \mathrm{C}, 65.59 ; \mathrm{H}, 3.90 ; \mathrm{N}, 15.94 ; \mathrm{O}, 7.28$; S, 7.30.

Found: C, 65.55; H, 3.92; N, 15.92; O, 7.30; S, 7.31.

Mol. Formula: $\mathrm{C}_{24} \mathrm{H}_{17} \mathrm{~N}_{5} \mathrm{O}_{2} \mathrm{~S}$

Mol. Wt.: 439.

6-b) ethyl2-cyano-2-(7-cyano-2-(4-methoxyphenyl)-6-oxo-4phenyl-4, 6, 9, 9a-tetrahydropyrimido $[2,1-b][1,3]$ thiazin-8yl)acetate:

IR: 3330, 2210, 1650, 1050, 2910, $1710 \mathrm{~cm}^{-1}$;

${ }^{1} \mathrm{H}$ NMR: $5.08(\mathrm{~s}, 1 \mathrm{H} \mathrm{NH}), 6.44(\mathrm{~s}, 1 \mathrm{H}=\mathrm{CH}), 5.76(\mathrm{~s}, 1 \mathrm{H} \mathrm{CH})$, 5.51 (s, 1H CH), 7.27 (s, 5H Ar-H), 6.89 (dd, 2H Ar-H), 7.22 (dd, 2H Ar-H), 3.98 (s, 1H act.-CH), 3.79 (s, 3H), 4.19 (q, 2H), 1.22 (t, $3 \mathrm{H})$.

ESI-MS: 486.

Anal. Calcd: $\mathrm{C}_{26} \mathrm{H}_{22} \mathrm{~N}_{4} \mathrm{O}_{4} \mathrm{~S}$ : C, 65.77; H, 4.42; N, 12.27; O, 10.51; S, 7.02 Found: C, 65.74; H, 4.44; N, 12.28; O, 10.53; S, 7.01.

Mol. Formula: $\mathrm{C}_{26} \mathrm{H}_{22} \mathrm{~N}_{4} \mathrm{O}_{4} \mathrm{~S}$.

Mol. Wt.: 486.

\section{Acknowledgements}

The authors are grateful to Dr. G. N. Shinde, Principal, Yeshwant Mahavidyalaya, Nanded. For providing laboratory facilities and Vishnu Chemical Ltd, Hyderabad, for providing spectral data.

\section{References}

[1] H. Joshi, P. Upadhyay, A. J Baxi, J. Indian Chem. Soc., 67, 779 (1990).
[2] S. P. Rathod, A. P. Charjan and P. R. Rajput, Synthesis and Antibacterial Activities of Chloro-Substituted-1, 3-Thiazines, Rasayn J. Chem., 2010.

[3] C. Sanjeeva Reddy and A. Nagaraj, Synthesis and Biological Study of Novel Bis-chalcones, Bis-Thiazines and Bispyrimidines, J. Iran. Chem. Soc., Vol.5, No.2, June 2008, 262267.

[4] Mamoru Kokestu, Kohsuke Tanaka, Yuichi Takenaka, Cecil D, Kwong and Hideharu Ishihara, Synthesis of 1, 3-thiazine derivatives and their evaluation as potential antimycobacterial agents, European j pharm. Sci., April 2002, volume 15, Issue 3, Pg. no. 307-310.

[5] R. Kalirajan, S. U. Sivakumar, S. Jubie, B. Gowramma and B. Suresh, Internl. J. Chem Tech Res., Jan -March 2009, Vol 1, No.1, 27-34.

[6] Tojkoporan, Synthesis of some 1, 2, 4-triazolo [3, 2-b]-1, 3thiazine-7-ones with potential analgesic and anti-inflammatory activities, pubmed, $2002 \mathrm{Feb} ; 57$ (2), 145-52.

[7] Li Fu, Li Fu, Ding Ye, Ying Li and Shufan Yin, Synthesis and claiming activity of 6H-2-Amino-4-Aryl-6-(4-b-DAllopyranosyloxyphenyl)-1, 3-thiazine, Chemistry of natural products, vol.46, no.2, 169-172.

[8] Zawisza, Synthesis and pharmacological analysis of new derivatives of tetrahydro-[1,3]-thiazine and 2-thiobarbituric acid, National center for biotechnology information, 1981; 29 (2), 235-48.

[9] Jin Guiyu Cao Chunyang, Synthesis and biological activities of substituted pyrazolo [4, 5 e] $[1,3]$ thiazine derivatives, Chinese journal of pesticide science, 1981; 29 (2), Pg. no.; 235-48.

[10] Diurno M V, Mazzoni O, Piscopo E, Calignano A, Giordano F, and Bolognese A 1992 J. Med. Chem. 352910.

[11] Rawal R K, Tripathi R, Katti S B, Pannecouque C and DeClercq E 2007 Bioorg. Med. Chem. 153134.

[12] (a) Ottana R K, Carroti S, Maccari R, Landini I, Chiricosta G, Caciagli B, Vigorita M G and Mini E 2005 Bioorg. Med. Chem. Lett. 15 3930; (b) Gududuru V, Hurh E, Dalton J T and Miller D D 2004 Bioorg. Med. Chem. Lett. 145289.

[13] Campaigne E and Nargund P K 1964 J. Org. Chem. 29224.

[14] Dana W. Mayo, Ronald M. Pike, David C. Forbes; Microscale Organic Labor-atory; with Multistep and Multiscale Synthesis, (2010) 308-310.

[15] S. V. Kostanecki, Tambor; J. Chem. Ber., 32, 1921 (1899).

[16] Jain AC, Prasad AK (1995) Reaction of chalcone 2-4,dimethoxychalcone, a-bromo and methoxy chalcone with thiourea. Indian J Chem 34B: 496. 14.

[17] Sambhaji PV, Vijay NB, Sandeep V. Khansole, Ramdas NK (2009) A convenient one pot synthesis of 3-cyano-9-methyl-2methylthio-4-0xo-4H-pyrimidi-[2, 1-b] [4, 5-b] quinolone and its reaction with selected nucleophiles. Letters in organic chemistry 6: 544-548. 